\title{
Validity of the EatWellQ8 online food frequency questionnaire against a 4-day weighed food record
}

\author{
B. Al-Awadhi ${ }^{1}$, R. Fallaize ${ }^{1}$, R.Z. Franco ${ }^{2}$, F. Hwang ${ }^{2}$ and J.A. Lovegrove ${ }^{1}$ \\ ${ }^{1}$ Hugh Sinclair Unit of Human Nutrition and Institute for Cardiovascular and Metabolic Research and \\ ${ }^{2}$ Biomedical Engineering, School of Biological Sciences, University of Reading, UK, RG6 6AP
}

The online EatWellQ8 (EWQ8) food frequency questionnaire (FFQ) was developed as a web-based tool for the collection of dietary intake for adults living in Kuwait ${ }^{(1)}$. Further validation against data derived from an independent and established tool is necessary to ensure its accurate assessment in both nutrient and food group intake ${ }^{(2)}$.

The objective of the study was to assess the validity of the EWQ8 FFQ against a 4-day weighed food record (WFR) in adults living in Kuwait. A total of 60 healthy volunteers (67\% female: $33 \%$ male) aged range between 18-65 years were recruited from Kuwait and were asked to complete a 4-day WFR 1-week after the administration of the EWQ8 FFQ, which included 146 food items and food portion size photos. The level of agreement between nutrient and food group intakes estimated by the EWQ8 FFQ and a 4-day WFR were evaluated using t-tests, Bland-Altman methodology and classification into quartiles of daily intake. Crude unadjusted correlation coefficients were also calculated for nutrient and food group intakes.

In total, 46 (63\% female: $37 \%$ male) participants completed the EWQ8 FFQ and the 4-day WFR (mean age 35, SD 8.4 years). Crude unadjusted correlations for the EatWellQ8 FFQ and the 4-day WFR ranged from (0.40-0.88). Mean cross-classification into "exact agreement plus adjacent" was $85 \%$ for nutrient intake and $83 \%$ for food groups. Bland-Altman plots showed good agreement for energy-adjusted macronutrient intakes (Fig.1 A-D).
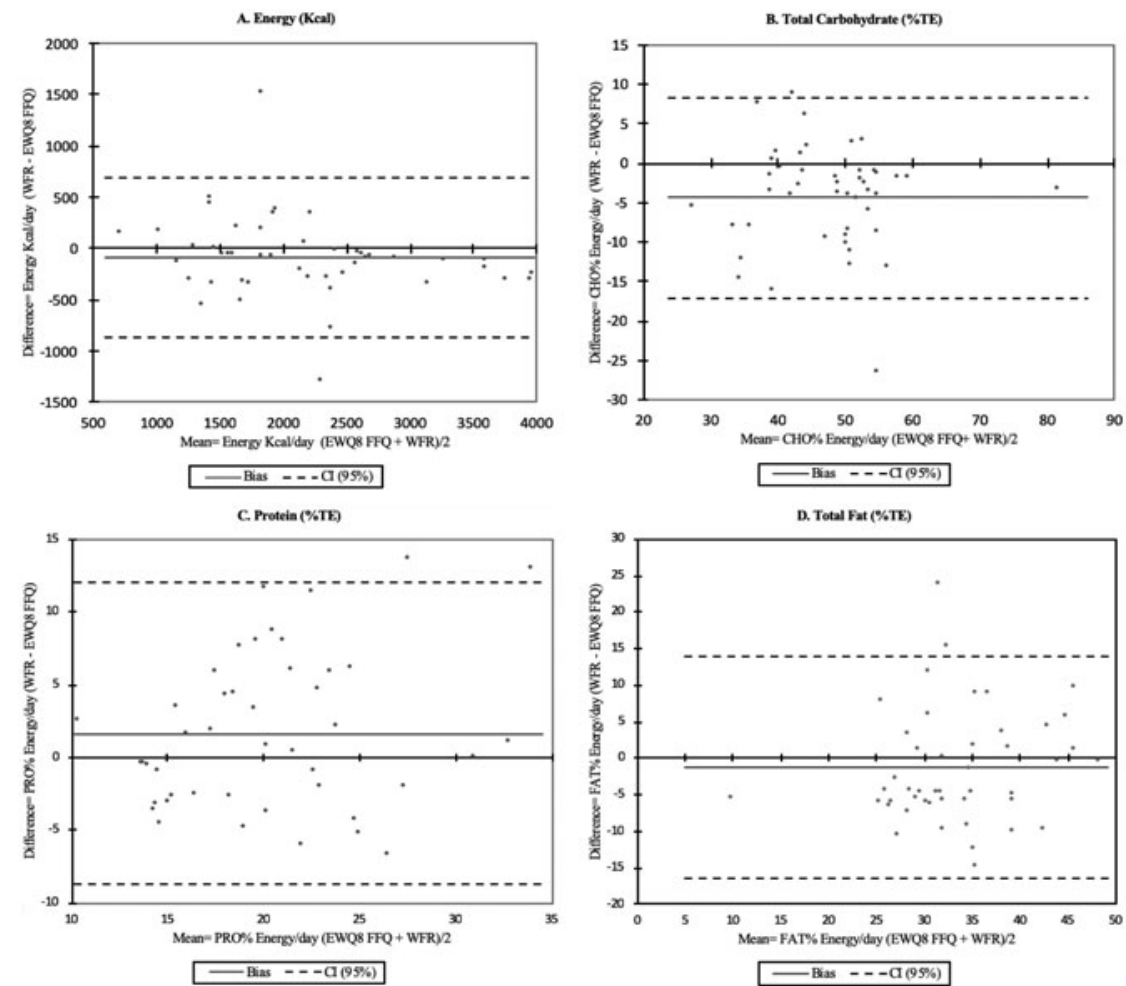

Fig. 1. Bland-Altman plots comparing the EatWellQ8 FFQ to a 4-day weighed food record for (a) energy, (b) total carbohydrate, (c) protein, and (d) total fat. The solid line represents the bias (mean difference) and the dotted lines represent the limits of agreement. \%TE: \% Total Energy

The online EWQ8 FFQ has good agreement with a 4-day WFR for assessing energy and energy-adjusted nutrient intakes. The EWQ8 FFQ is a suitable web-based tool for assessing dietary intake in healthy adults in Kuwait.

1. Franco RZ, Alawadhi B, Fallaize R et al. (2017) JMIR human factors 4.

2. Cade J, Thompson R, Burley V et al. (2002) Public Health Nutr 5, 567-587. 